\title{
THE BUSINESS ASSISTANT SERVICE AS ONE OF THE PROMISING AREAS FOR THE ADOPTION OF AI TECHNOLOGIES IN THE ENTERPRISE
}

\author{
Tetiana IVASHCHENKO ${ }^{1 *}$, Igor CHORNODID ${ }^{2}$, Andrii IVASHCHENKO ${ }^{3}$ \\ ${ }^{1,2}$ Acadamy of Labour, Social relations and tourism, Kyiv, Ukraine \\ ${ }^{3}$ Ciklum, Kyiv, Ukraine
}

Received 21 April 2020; accepted 15 June 2020

\begin{abstract}
In modern conditions, entrepreneurs are faced with the acute problem of analysing of numerous information, quickly responding to a constantly changing economic situation, and making the most optimal decisions. In this regard, the development of a Business Assistant service (BAS) is a very relevant since it is a modern solution that can significantly simplify and improve the work of enterprises. The main goal of the research is on the basis of AI technologies to elaborate the Business Assistant service, that would speed up, optimize and simplify the decision-making process for the entrepreneur and can be used by many enterprises both when starting a business and when operating it. The main tasks for implementing the goal are: to analyze the scientific literature regarding the possibilities of using AI technologies in business, to identify the factors that mainly influence the entrepreneur's choice regarding the sphere of activity, as well as the types of information most useful for doing business, to analyze and collect data for the model design, to develop a prototype of the BAS and test its functionality in practice. The research methods are: the theoretical - analysis and synthesis, abstraction method, the empirical - modelling (clustering, classification, logistic regression) and experimental method.

The investigation results are: a prototype of the BAS was created, its effectiveness - ability of delivering useful recommendations and improving the business decision-making process for the entrepreneur has been proven experimentally using actual market data. The service can be effectively used by small and medium-sized enterprises in various industries and regions, provided that there is an access to the necessary data. The main risks associated with its implementation and possible ways of their reduction were considered.
\end{abstract}

Keywords: business assistant service, artificial intelligence technologies (AIT), enterprise, business model, decision making process.

JEL Classification: C45, C55, C61, M15.

\section{Introduction}

Nowadays, computer programs created on the basis of artificial intelligence $(\mathrm{AI})$ technologies are widely used in the various spheres of human activity - logistics of transportation, medical services, banking, financial operations, industry optimization, autonomous driving, urban infrastructure, education and much more.

This is not surprising, because despite the relatively short period of practical use, artificial intelligence systems have been able to prove their effectiveness and are therefore a promising avenue for development and further use in the future.

But when it comes to the enterprise, which is an integral part of the economy, the driving force of important economic transformations and an indicator of the viability (health) of the national economy, in the future, it is really hard to imagine it as non-active user of innovative technologies in its daily activities. To become an enterprise of the future, one should undergo transformational change such as widespread introduction of developments in the field of information technology into its business processes.

These days, regardless of what business you are in, applications of AI, based on machine learning, can be found across the full range of business activities. This covers everything from document processing, automation of recruiting business processes, answering customer queries and target marketing through to robots on the production line, warehouse management and customer deliveries (Finlay, 2018).

Currently arises another perspective area - this is implementation of AI technologies into the process of business decision-making, that can greatly benefit and positively influences modern businesses (Kashyap, 2018).

${ }^{*}$ Corresponding author. E-mail: tanita.ivash@gmail.com 
The main problem is that modern enterprises with each day have to deal with a bigger variety and volume of data and large amounts of information, needed to be analyzed. Since physical and human resources are limited, taking into account all the nuances and factors affecting the final decision is often very difficult and even impossible task. In this regard, it is necessary to expand the staff, attract new employees, establish expert departments. All this significantly increases the enterprises' expenditures, especially this is true for small firms, where financial opportunities are often limited.

Another not less important problem is that it takes a lot of time to analyze numerous information and the decision that will be made later may not be already relevant and will delay the entrepreneurs timely reaction. As a result of this, entrepreneurs can lose potential customers, suffer losses and even leave businesses.

In addition it is known that many people who start their own business often do not even know what they would like to do, what type of business is promising and brings a steady income at a given time. Without access to such information, many people are doing the wrong thing and as a result they often suffer losses, leave business early without getting the desired profit. And even if the enterprise already exists, untimely access and analysis of information on product prices, changes in legislation, credit conditions can directly affect the operation of the enterprise and its financial stability.

It has been proven, that products based on AI technologies can process a lot of data at once and handle many different factors simultaneously. As a result of better computational processing and cheaper data storage solutions they are able to issue much better results and provide users with the best possible decisions (Kopanakis, 2019).

Considering all mentioned above and given the fact that the viability and profitability of an enterprise often depends on the timely and correct decision-making, the main goal of our research was to introduce a technical solution that would be like virtual assistant to the entrepreneur (Business Assistant Service), and will be able to help him both at the stage of creating an enterprise, choosing the field of activity and further on when running a business and managing the enterprise. The creation and implementation of Business Assistant service is very actual, since it would greatly speed up, optimize and simplify the decision-making process for the entrepreneur.

The idea of creating an advisor is not totally new. In order to improve traditional customer service with AI, some insurance companies are using "robot advisors", that are algorithms which on the basis of analyzed information can provide automated financial advice for consumers as to which insurance package would be more suitable and profitable for them (OECD, 2017). But while robo-advisor is oriented on customers, satisfaction of their needs, the service we offer is targeted on the entrepreneur and can be used in enterprises of many types in order to track important for the entrepreneur information and not to miss anything that could be useful when starting a business and keeping it afloat.

To achieve the goal set, at the first stage, we determine the factors that mainly influence the entrepreneur's decision-making regarding the choice of the business sphere, one would like to work in, then we distinguish the main types of information that would be useful for the decisionmaker in the process of running a business. At the second stage, based on the use of artificial intelligence technologies, a Business Assistant model will be introduced and an example of its practical application will be shown.

The material of the study is structured in the following way: firstly, we provide a short review of the economic literature on the main prospects and problems of the implementation of the Artificial Intelligence technologies in business; secondly, we give description of the process of developing a Business Assistant model using theoretical and empirical research methods, then we present the main results of the study, test the model, and finally - draw conclusions and outline the main concerns we faced.

\section{The theoretical analysis of the research}

The importance of AI and expert systems, which are designed to help entrepreneurs and managers in decision making is undisputable. This can be understood from the increased interest of scientists and the emergence of new scientific publications devoted to this issue. In order to assess the current perception by scientists of the problem of using AI technologies for the improvement of the business decision-making process the latest scientific literature sources were analyzed.

In the course of the research, it was found that many scientists pay significant attention to the popularization of AI technologies among the different business organizations, focusing on the effectiveness of its use in the improvement of the decision-making process by entrepreneurs. At the same time some of the scholars emphasize on the risks and problems associated with the widespread adoption of IT in the enterprises' work processes, their immaturity and limitations.

The most common view of the problem is that, despite the fact that the use of AI technologies for business decision making still remains a relatively unexplored area, it is obvious that they can positively influence the last and is able to simplify the process of decision making and give entrepreneurs and managers a valuable support and guidance. AI programs can provide user with quicker, more thoughtful and original decisions, handle multiple inputs, in addition they don't get tired (Kopanakis, 2019). This statement can be supported by the current works of Lithuanian researchers as they assert that usage of $\mathrm{AI}$ in decision making and Quality Management Systems reduces information overload, shortens decision making time and human workload on the information processing. But in order to perfect the quality of AI Management System and not to mismanage freedom, human emotional experience 
both rational and creative decision making models should be harmonized (Paliukas \& Savaneviciene, 2018).

Trying to understand the challenges and opportunities associated with the use of advanced technologies in the business decision-making processes other scientists have also analyzed that modern expert systems are generally able to solve complex business problems and come up with a right decision but there could be gaps between development of the expert systems and real life processes. They did a good attempt at development of the decision making model, based on the application of Artificial Neural Networks and Particel Swarm Optimization algorithm, that could be used in decision support systems e. g. investment or credit management (Neortaite \& Bulteris, 2009).

Present-day entrepreneurs and managers are aware that making effective decisions is different from just having excellent knowledge and access to the necessary information. AI technologies, which have already proven themselves in the ability to learn to recognize patterns in data sets subject to the constantly changing conditions, including market ones, should be actively used in the transition from traditional to the fundamentally new, intellectually trained decision-making processes (Michalewicz et al., 2006). Of course the decision maker remains the most important part of the decision-making process, but the potential and the need of AI expert systems' implementation in the work of enterprises is an important condition for improving the quality and simplicity the decisionmaking process by a responsible person (Phillips-Wren \& Jain, 2012).

Furthermore, since sophisticated neural network techniques such as language processing, deep learning, graph theory and others, using vast quantities of data, are able to conduct a qualitative analysis of the information received from various sources. This possibility is widely used by financial companies for the development of the effective AI solutions that are capable to improve and facilitate the decision-making process, which in turn contributes to the development of the company, the increasing of its competitiveness and sustainability. A group of AI experts from the OECD have concluded, that in connection with the rapid development of information technology, a new type of business is emerging called e - business, where timely access, fast and high-quality analysis of information becomes a priority for any enterprise and allows one to reduce costs and increase profits (OECD, 2019).

This statement can be supported by the works of some progressive economists, which assume that AI will soon affect organizations in every sector (Schatsky et al., 2014). Since machine learning is able to draw conclusions from large and complex data sets, they may be helpful in making high-quality predictions from operational data which is critical for modern companies because it can significantly improve enterprises performance, increase revenues and create new capabilities, enhancing their competitive position on the market (Schatsky et al., 2015).
At the same time some scientists are not so optimistic and distinguish the risks and problem associated with the in the widespread involvement of AI technologies in decision making processes.

Despite of the high expectations from AI programs, business adoption of them is almost not implemented because of a disparity between expectations and actions. Therefore, the use of high-tech decision-making programs is not urgent for many companies today as entrepreneurs and managers often choose other priorities like competing investment or unclear business cases (Ransbotham et al., 2017).

In addition there is no common perception and understanding of $\mathrm{AI}$ and its positive effect on the qualitative improvement of the management decision-making process. This bears fundamental obstacles to the implementation and active use of modern AI technologies in practice and also in turn inhibits further researches in this field (Bawack et al., 2019). Although There are also other substantial challenges of AI technologies adoption for business decision making, for example, it is hard to integrate cognitive projects with existing processes and systems, AI technologies are too expensive, managers often don't understand how technologies work and finally technologies by themselves are immature (Davenport \& Ronanki, 2018).

Finally, there are some risks around the use of AI technologies in the business processes including decision making ones (Shefford \& Holland, 2018). They believe that since advanced technologies become ordinary for many organizations there is a real threat of loosing control over managing the risks effectively or providing guarantees of stable company's functioning. Authors insist on asserting sufficient control over the technology in order to avoid consequences like cyber-attacks, errors coming from wrong learning, destructive effect on the corporate culture, untimely and poor-quality elimination of the system operation errors due to the lack of qualified personnel aware of the system functioning peculiarities, unreliability of decisions made by AI systems.

It should be mentioned, that most of the studies are aimed at a general understanding of what advantages and disadvantages are from embedding and using the AI technologies in order to improve the decision-making process in modern businesses' operation. But there are only few of them which are devoted to the development of new ideas and new products that can have practical application and the use of which can increase the efficiency of the decision-making process by modern entrepreneurs. Therefore, our goal was to make such a offer and thereby expand the field of application of AI technologies in business.

\section{Investigation methodology}

During our investigation several research methods were used. Among them - theoretical research methods (anal$y$ sis and synthesis, abstraction method); empirical methods - modelling (clustering, classification, logistic regression) and experimental method. 


\subsection{Theoretical research methods}

The development of the artificial intelligence systems is based on the analysis of a large amount of data. For the most effective system training the more data available, the more accurate the model is. In our case, for the fullfledged operation of the system, it is necessary to collect data on the vast majority of enterprises, and ideally, all enterprises located in this geographical location.

But, in practice, this is not possible. In the most of the countries, this information is considered confidential, especially information about the profits. Also there is a lack of unified mechanisms for providing and collecting this information. According to the legislation of Ukraine, this information must be open (Law of Ukraine, 2017). But, as practice shows, getting this information even after the taking effect of this law is not possible (Ivankin, 2018). For the full functioning of such systems as Business Assistant it is necessary at the legislative level to allow the use of such information. However, this possibility entails certain risks. The main risk is that with the open access to confidential data arises the possibility of using this information for unlawful purposes. To prevent this we would offer to provide the information requested through special services ensuring control and safety of this data, as well as providing only those data, which is necessary for calculations, without disclosure of the enterprises confidential information. As one of the options we can offer the system similar to Sweden, where there online information of income of any citizen is available (Weller, 2017). The next risk is the possibility of getting false information into the system. Business Assistant is self-learning system and its learning depends on the data collected from different sources. Getting into the system of unverified or false data may lead to a decrease in the accuracy of the software service. To avoid this, it is necessary to ensure control over the correct functioning of the system, as well as to envisage the possibility of returning the system to its previous state, thereby leveling the wrong learning on the incorrect data. In addition, given the fact that the Business Assistant is the system that is constantly training on the incoming data, the issue of monitoring the correct functioning of the model is a very important. This problem can be solved in several ways, from the periodic checking of the system's functioning manually to the creation of automatic tests that will check the system at specified intervals.

Because getting full information is not possible at this time of the moment, for training the Business Assistance model we used data from open sources to train the system and took the financial statements of 3,000 companies located in the USA - small and medium. (The Open Data of 500 US companies, 2020; Fortune 500, 2019; Inc., 2019).

The main attributes that were used were: location, size of the enterprise (small, medium, large enterprise) and income.

To train and test the RBA system, we used widerange real-time data such as stock quotes, exchange rates, prices of goods, raw materials, services, other, as well as legislative changes related to conducting business.

To obtain this information we used such open sources: (The Billion Prices Project, 2019; Our World in data, 2020; ActiveTrades, 2020; US Stocks, 2020; Etrade, 2020; Investing.com, 2020; OANDA, 2020).

Collection of all this information was done by manually gathering and using special programming scripts. Acquired data was deeply analyzed, normalized, categorized and split to the suitable for ML process datasets. During the research we determined and created more than 200 categories based on these datasets, such as areas of activity of companies, for example, agro, software, construction, food and beverages, etc, as well as more detailed ones: prices for grain, vegetables, fruits, oil, oil products, building materials, etc. In addition, we used open sources of information on financial institutions credit offers, as well as information from the websites of government agencies about changes in legislation regarding business operation, taxes, etc. (US government, 2020).

Abstraction method was used in order to distinguish factors that commonly affect the entrepreneur's decision making while selecting a particular field of activity when starting a business. Many authors (Soumya, 2019; Hanlon, 2015) identify from 7 to 10 factors, for example, scale of the business, capital requirement, degree of risk, business stability, system of management, profit division.

Such factors as degree of risk, business stability, can be combined together according to a common feature geographical location. Because in one geographical location, these parameters are usually the same. At the same time, capital requirement and scale of the business can be combined into one parameter, such as the size of the enterprise. This optimization allows us to reduce the number of the system parameters and make the process of collecting information and training the model easier.

After this optimization we selected the three factors that mainly influence the choice of entrepreneurial activity direction (area):

- location - the geographical location, where it is planned to open an enterprise (this may be a specific locality, region / state, country);

- size - size of the enterprise, which one plans to open. The Ministry of Finance of Ukraine uses such criteria as the book value of assets, net income and the average number of employees to divide enterprises into small, medium and large (Voitenko, 2019), U.S. Small Business Administration uses these criterias: ownership structure, number of employees, earnings and industry (Liberto, 2019), but the majority of the countries use two from three criteria (revenues, assets or a number) for this separation (Kenton, 2019). That is why, the SBA uses an internal ratio to divide enterprises into small, medium and large, based on annual profits and the number of employees. Service Business Assistant will operate for small and medium enterprises. Large enterprises are usually corporations, multi-directional firms, etc., which have their own business analytical departments, and 
which usually contain a staff of high skilled experts able to help in determination of the corporation future development strategy. Although, even for such enterprises, the Business Assistant will be useful as an auxiliary tool for business analysts and experts.

- profitability - enterprise quarterly profitability. Unfortunately, at the moment it's not possible to collect information on the quarterly profitability of enterprises, therefore we used information from open sources where only annual or three-year profitability is provided.

These are the main criteria that affect the choice of entrepreneurial activity.

\subsection{Empirical research methods.}

Using the modelling method, a prototype of Business Assistant was created. Two main services are implemented. The first service (Starting Business Assistant, SBA) is used at the stage of creating an enterprise and registering it with government agencies. The second service (Running Business Assistant, RBA) is used to support an already operating enterprise, help in carrying out business, determine the direction of further development, as well as inform about the possibilities of profitable financing / lending.

The Figure 1 in addition to the already mentioned SBA and RBA shows the following:

1. Financial statement API - a module that collects data of the financial statement of companies from certain sources. The received data is being sent to the Data Processing Module.

2. Data analysis system (ML Processing module) ML service, which produces model training based on the received data from the Data Processing Module. It provides results based on the received data, in response to the SBA and RBA requests.

3. Distributed analyser of additional data, DAAD - is a service that collects from open sources special information, such as changes in the price of goods, in stock quotes, in legislation, offers from financial institutions for lending products, etc. It sends results to the ML Processing module. This information is used during the processing requests from RBA.

4. User interface - UI - interface that allows users communicate with Business Assistant. Depending of the user needs UI makes requests to SBA or RBA.

The Figure 1 demonstrates communication flow between these modules. Users via UI make request to SBA or RBA, which in turn communicates with Data analysis system and return results to UI. Data analysis system collects information from Financial statements API via Data processing module and additional information from DAAD.

Starting Business Assistant requires that user should register in the system, enters these data: the location of the business, the size of the company, which is planned to open, and the estimated income. The Business Assistant system processes this data and displays in the descending order types of entrepreneurial activities in the specified geographical location that in case of their choose would be the most profitable for this user. Also Business Assistant offers a list of documents and references, necessary for the business opening at a given location.

RBA works differently. After registration user selects the areas of his entrepreneurial activity, and categories of alerts he interested in, for example, changes in the prices of goods in different regions of the country, changes in the prices for raw materials, loan offers, etc. RBA collects additional information from open sources using an internal service Distributed analyzer of additional data (DAAD) (see Figure 1), such as information on changes in prices for goods, raw materials, changes in legislation, financial proposals from banks, etc. This information is processed by the ML processing module, that determines which target group is interested in it. RBA notifies users according to their subscription categories when the system recognizes that the information received is useful for this category of users. In addition, for training the RBA classifier, the system will suggest users to indicate whether this

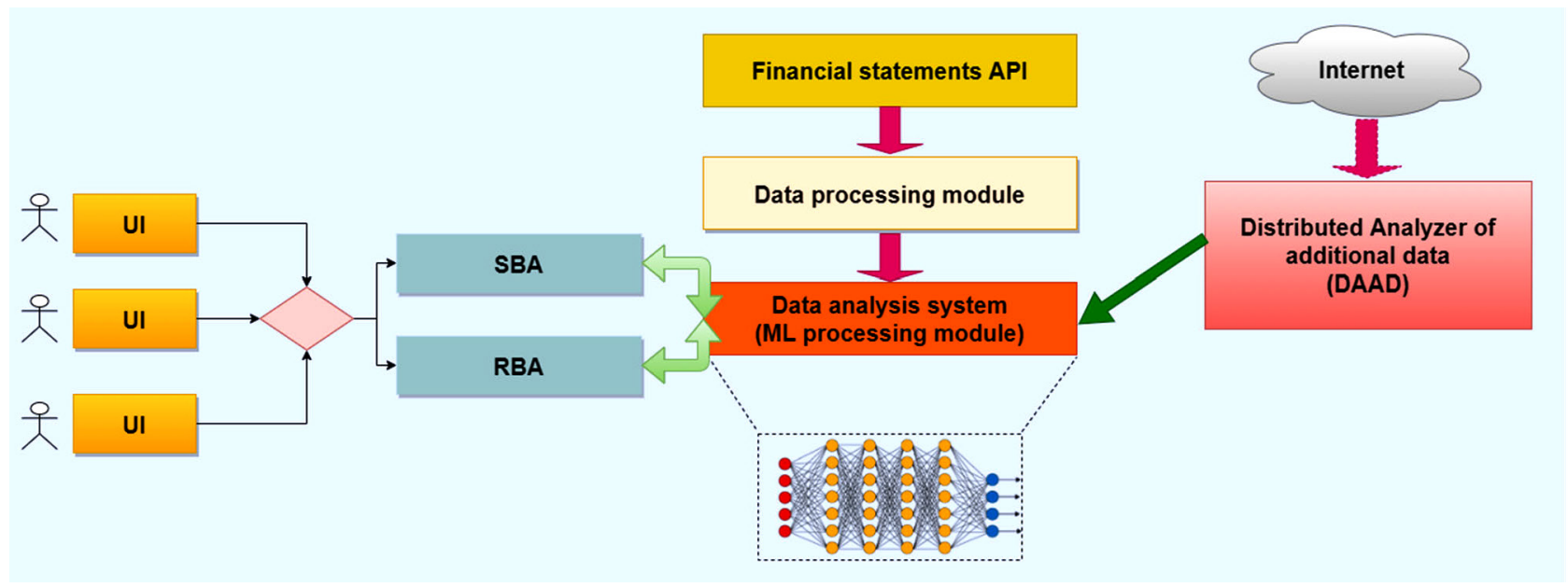

Figure 1. The high-level architecture of the Business Assistant application 
information was useful to them. Based on the feedback received, for more accurate users' separation into target groups, clustering adjustment will be conducted.

During the modeling of Business Assistant, we used these methods:

1. Classification;

2. Clustering;

3. Logistic regression.

Classification method was widely applied for the modeling the Business Assistant. In the SBA there are two Classifiers (see Figure 2), first is to determine which cluster the particular company is closest to, and second for measuring the types of entrepreneurial activities that are relevant to the user's request. For implementing these classifiers Random Forest and logistic regression algorithms were used. The RBA service also uses two classifiers. They are - verification classifier, which determines whether the information received is sufficient for users notification, and determination classifier, that determines which categories of users will be interested in this information. Verification classifier uses logistic regression. The main parameters of it are the subject of information (for example, the price of grain), the price - the previous one, and the new, geographical location, source of information, volumes. Determination classifier is based on Random Forest Algorithm.

Clustering is used during the modeling of both services of the Business Assistant - SBA and RBA. For SBA unsupervised clustering learning algorithm Hierarchical Cluster Analysis (HCA) applies to divide data from datasets into clusters. For this process the following attributes are used: location, expenses, income and size of the enterprise.

In RBA the Hierarchical Cluster Analysis is used for clustering users into groups.

Logistic regression - In SBA and RBA logistic regression is used as a base method in the classifiers. In SBA it is used in Classifier, see Figure 2, that determines which cluster the user most likely belongs to. And in RBA it is base of the Verification classifier (see Figure 2) which determines whether the information received is sufficient enough to notify the users.
In Figure 2 the mechanism of the processing information in Business Assistant is presented. In SBA the initial training dataset is split into the clusters using Hierarchical Cluster Analysis algorithm. For combining new company data with existing clusters, we created and trained classifier based on Random Forest algorithm. Another classifier grounded on logistic regression is used for finding clusters, which most closely match to the user's request. This classifier communicates directly with SBA input/output system. In RBA real time data is analyzed by verification classifier. If it detects that this information is important, it sends it to determination classifier, which determines which user groups need to be informed about this.

In order to prove the effectiveness and reliability of the Business Assistant model the practical experiment was conducted. Its essence is to assess the accuracy of the service results based on real data.

To test SBA, we have collected data about 3,000 US companies from open sources. We used the following attributes: location, size of the enterprise (small, medium, large enterprise, a special coefficient was used to determine it, based on revenue and the number of employees hired), revenue. We divided the geographical location into several attributes - the first is the state, and the second and third are longitude and latitude. For creating this module we used artificial intelligence framework Scikit learn. We split the original data set into 270 clusters using the Density-based spatial clustering of applications with noise (DBSCAN) algorithm.

As an experiment, we decided to check what recommendations will Business Assistant provide for this test case: Location - US, Texas, city - Austin; company size small; Desired profit - 3-5 million US dollars.

In order to test $R B A$ have prepared the dataset of 10,000 events contained such information as: changes in prices for goods, services, stocks, as well as changes in US law regarding business regulation for 2019, 2020.

Data was first tagged using the unsupervised learning algorithm Isolation forest. Also, part of the data was processed and tagged manually.

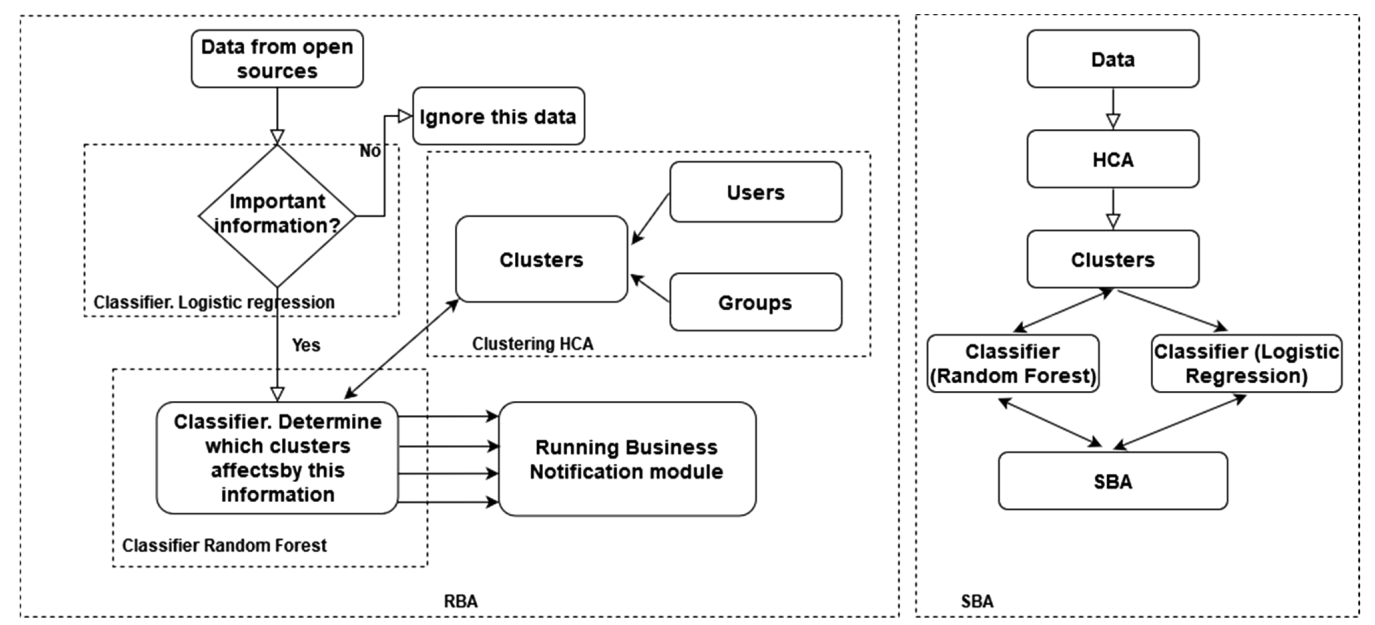

Figure 2. Schema of the processing information in starting business assistant and running business assistant 
For the experiment Running Business Assistant had been running in the test mode for 30 days period, from January 8, 2020, to February 6, 2020. For validation the correctness of RBA, all messages from the system were analyzed and compared with the current market situation.

To simplify the experiment, only three parameters were taken into account:

- changes in exchange rates;

- changes in the price of grain in the US;

- changes in US law for the current period.

\section{Results and discussions}

In the course of an experimental study, the following results were obtained.

\subsection{Results and discussions for SBA}

As described above to check the accuracy of the SBA service we have prepared special datasets. Using the Random permutation method, we split this clustered data set into "test set" and "validation set" in the proportions of $80 \%$ to $20 \%$.

After training the SBA on these datasets, we used Cross-Validation method as a performance metrics of the obtained result.

For training set using this formula we got such values:

$$
\text { crossValScore }\left(c l f, X_{\text {train }}, Y_{\text {train }}, c v=3 \text {, scoring = accuracy }\right) \text {, }
$$

$\operatorname{array}([0.9302,0.94565,0.93495])$. This means, that with probability more than $93 \%$ SBA recognizes correct profitable business areas on the training set.

For validation set (data that was never seen for the system before), with formula (1) we got the following values: $\operatorname{array}([0.9214,0.92768,0.92657])$. So, it means that with the probability more than $92 \%$ (0.9252) SBA recognizes correct profitable business areas on the data, that was not analyzed previously during the training. Thus, SBA with a high degree of probability offers the most profitable business areas.
As a result of the experiment described in the section "Experiment for SBA" we got such results (see Table 1).

Table 1. Display of top clusters according to our request, areas of these clusters and final propositions from Business Assistant for the test example

\begin{tabular}{|c|c|c|}
\hline $\begin{array}{l}\text { Cluster } \\
\text { Number / } \\
\text { Probability }\end{array}$ & $\begin{array}{l}\text { Top areas of this } \\
\text { cluster (global } \\
\text { categories without } \\
\text { detail) }\end{array}$ & $\begin{array}{c}\text { Suggested types } \\
\text { of entrepreneurial } \\
\text { activities by Business } \\
\text { Assistant }\end{array}$ \\
\hline \multirow{3}{*}{$\# 12-0.85$} & $\begin{array}{l}\text { Consumer Products } \\
\text { \& Services }\end{array}$ & \multirow{4}{*}{$\begin{array}{l}\text { Distributing } \\
\text { consumer goods } \\
\text { from a number of } \\
\text { industries across the } \\
\text { US }\end{array}$} \\
\hline & Software & \\
\hline & Health & \\
\hline \multirow{3}{*}{$\# 144-0.1$} & Health & \\
\hline & Education & \multirow{5}{*}{$\begin{array}{l}\text { Software, i.e. } \\
\text { developing an } \\
\text { asset tracking and } \\
\text { management platform } \\
\text { or evaluating financial } \\
\text { performance based } \\
\text { on ML }\end{array}$} \\
\hline & Manufacturing & \\
\hline \multirow[b]{4}{*}{$\# 221-0.05$} & Software & \\
\hline & Real Estate & \\
\hline & & \\
\hline & $\begin{array}{l}\text { Consumer Products } \\
\text { \& Services }\end{array}$ & $\begin{array}{l}\text { Health, providing } \\
\text { wellness services } \\
\text { across or training and } \\
\text { resources for medical } \\
\text { professionals }\end{array}$ \\
\hline
\end{tabular}

To check the suggestions from Business Assistant, we checked all the calculations manually.

Consider a test case. We introduced the desired profit of 3-5 million USD, we also chose a small enterprise. As a localization, we chose Texas, the city of Austin. We selected a list of small Texas-based companies in descending order by profitability. Top 5 companies from this list that meet our requirements are (see Table 2).

If we check the results of the experiment, described in the "Experiment for SBA" section, we can make conclusion that the recommendations made by the Business Assistant regarding the areas of "Consumer Products \& Services", "Software" and "Health" are correct.

Table 2. Top-5 small Texas-based companies with revenue 3-5 million USD and the largest profit growth in 3 years

\begin{tabular}{|l|c|c|c|l|}
\hline \multicolumn{1}{|c|}{ Company } & Revenue & Three-year growth & Location & \multicolumn{1}{|c|}{ Field of activity } \\
\hline Sky Marketing Corporation & $\$ 4.7 \mathrm{M}$ & $4,280 \%$ & Texas, Austin & $\begin{array}{l}\text { Distribute consumer goods from a } \\
\text { number of industries across the US }\end{array}$ \\
\hline Asset Panda & $\$ 5.1 \mathrm{M}$ & $3,779 \%$ & Texas, Frisco & $\begin{array}{l}\text { Software, Developes an asset tracking } \\
\text { and management platform }\end{array}$ \\
\hline Restore Hyper Wellness & $\$ 5.1 \mathrm{M}$ & $3,031 \%$ & Texas, Austin & $\begin{array}{l}\text { Health, Provides wellness services } \\
\text { across the nation. Services include } \\
\text { stretch therapy, cryotherapy, etc.. }\end{array}$ \\
\hline Owens Recovery Science & $\$ 5.4 \mathrm{M}$ & $2,657 \%$ & $\begin{array}{l}\text { Texas, } \\
\text { San Antonio }\end{array}$ & $\begin{array}{l}\text { Health, Provides training and resources } \\
\text { training and certification }\end{array}$ \\
\hline Valify & $\$ 3.1 \mathrm{M}$ & $2,276 \%$ & $\begin{array}{l}\text { Software, helps to evaluate financial } \\
\text { performance and reduce purchased } \\
\text { services costs, using ML }\end{array}$ \\
\hline
\end{tabular}


These obtained results allow us to conclude that SBA can be successfully used by small and medium enterprises, because such enterprises mainly operate in the one region and in the one economic sector, which in turn increases the accuracy of the SBA prediction. SBA can be applied at enterprises in various sectors of the economy and regions, for which there are sufficient initial data in the public access (size of enterprises, their scope, profitability, etc.). The SBA implications for practice are that it allows to choose more efficiently and reasonably the field of the activity at the stage of opening the enterprise, thereby having a beneficial effect on the economy by increasing the likelihood of the successful functioning of the new enterprises. The theoretical value of SBA is that we used the parameter "location" as an aggregation for a whole range of microclimate parameters of the economy, and we also successfully applied the combination of the Hierarchical Cluster Analysis (HCA), Random forest and Logistic regression algorithms to build the SBA prediction model.

\subsection{Results and discussions for RBA}

The same is for RBA, we split this data set into "test set" and "validation set" in the proportions of $80 \%$ to $20 \%$.

For the training set, we got the following values:

Using formula (1) we got these values: $\operatorname{array}([0.9745,0.9854,0.69792])$. As a result, we got accuracy about $98 \%(0.9797)$ on the training set.
For validation set, we got the following values using formula (1): $\operatorname{array}([0.9701,0.9727,0.9753])$. So, we got accuracy about $97 \%$ (0.9727) on validation set.

It means that with probability more than $97 \%$ RBA recognizes real time events, which require attention from users of BA.

Analyzing the statistics manually, we can see that the RBA correctly notified user about the important events and correctly clustered users.

The following are the graphs of the dynamics of changes in the exchange rate (USD to EUR) and grain prices in the period from January 8 to February 6, 2020. Figure 3 and Figure 4 show the graphs of exchange rate and grain prices with points, where RBA detected anomalies (red dots).

There were no legislative changes during the specified period, therefore, there were no notifications from the RBA about this.

Analysing these graphs and the messages received from the RBA, we can say that the system with a high probability notifies users about the events that are important to them.

The results obtained during the experiment allow us to conclude that the RBA with high accuracy recognizes the real time information that is important for conducting business, as well as determines the enterprises which this information will be most relevant for. The RBA implication

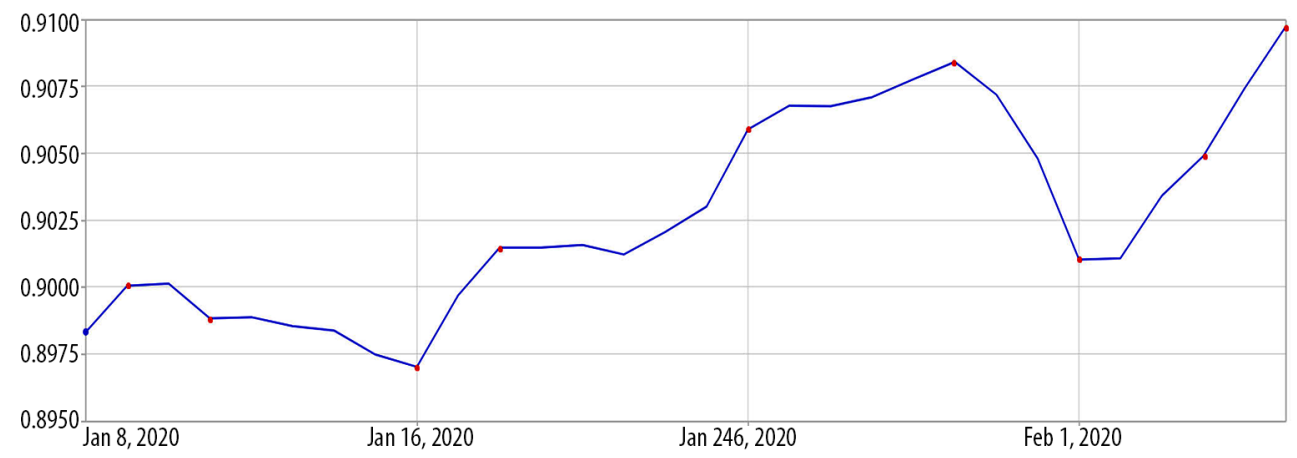

Figure 3. Graph of RBA anomalies detection in the exchange rate (ratio of USD to EUR) for the period from January 8 to February 6, 2020

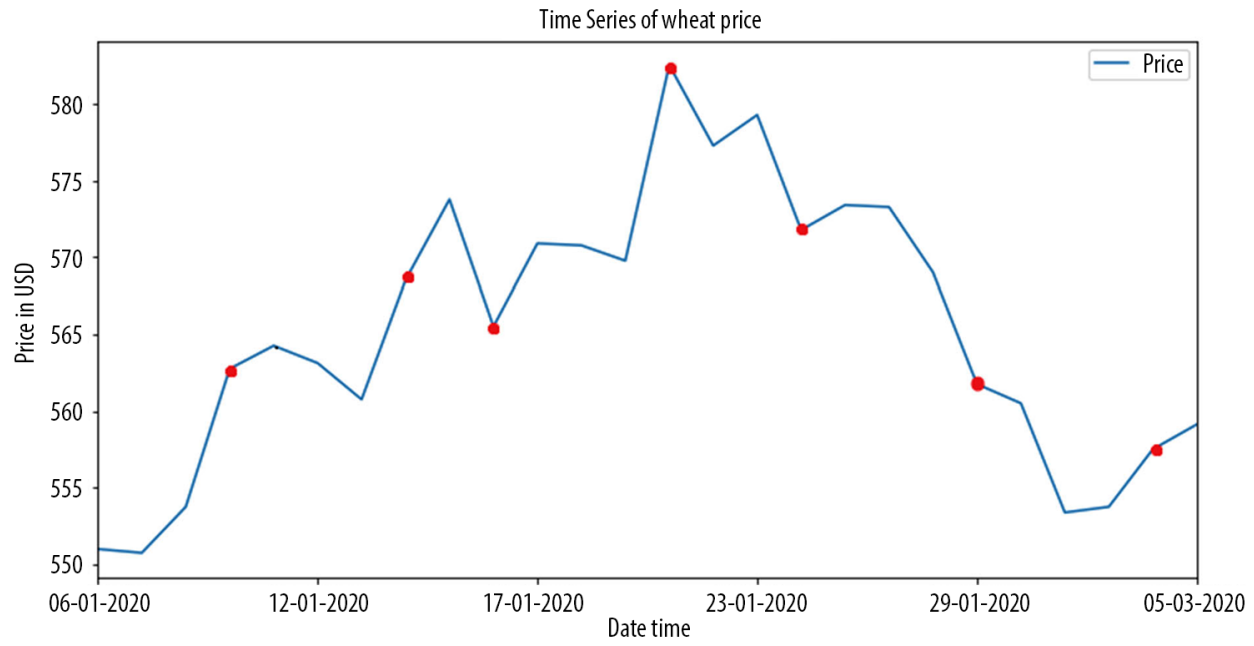

Figure 4. Graph of RBA anomalies detection in the grain prices for the period from January 8 to February 6, 2020 
for practice is of the great importance, because it allows small and medium-sized enterprises quickly respond to the market changes thereby increasing business productivity and profitability. RBA can considerably improve the work or even replace the analytical departments, that will have effect of the costs reduction and increase in speed and quality of the decision making process.

\section{Conclusions}

The rapid development of the economy and new technologies shows, that the use of systems based on ML and $\mathrm{AI}$ technologies in business operation is very relevant at the moment. During this study we managed to theoretically model and practically test the Business Assistant online Service, that can be an example of the use of advanced artificial intelligence technologies in modern business.

The main findings of the study are:

- Using the experimental method and relevant data, the efficiency and high accuracy of the predictions of the developed product "Business Assistant Service" was proved. The service is able to provide users with timely and correct recommendations on areas of business when opening an enterprise, continuously monitor and notify users regarding diverse market changes, like prices for goods and services, exchange rates, etc., as well as changes in legislation in the field of entrepreneurial activity and favorable offers from financial companies.

- Both component parts (Starting Business Assistant and Running Business Assistant) of the BAS can be mainly used by small and medium enterprises and be applied in businesses from various regions and sectors of the economy for which there are sufficient initial data in the public domain. The exceptions are state-owned strategic enterprises.

- The key to the effective functioning of the Starting Business Assistant service and obtaining more accurate results is open access to the necessary data, such as business activity area, its location, annual profits, number of employees etc. That is why it can be mainly applied in those countries, where it is available, for example, in USA, but not in Ukraine at the moment. For the full functioning of such systems as BAS it is necessary at the legislative level to allow the use of such information.

- The practical value of the BAS is that it allows entrepreneurs more efficiently and reasonably choose the field of activity at the stage of opening the business, thereby having a beneficial effect on the economy, increasing the likelihood of the successful functioning of new businesses. It allows small and medium-sized enterprises quickly respond to the market fluctuations, reducing analytics costs and increasing business productivity and profitability.

- The theoretical value of SBA is that it used the "location" parameter as an aggregation for a whole range of microclimate parameters of the economy, and also successfully applied the combination of the Hierarchical Cluster Analysis (HCA), Random forest and Logistic regression algorithms to build the SBA prediction model.

- During the study, it was also found out that although there are risks associated with the open access to the information, they can be reduced by ensuring control over the correct functioning of the system, as well as envisaging the possibility of returning the system to its previous state, periodic checking of the system's functioning manually or creating the automatic tests that will check the system at specified intervals.

- In future more research has to be done to develop the mechanism of using the Natural Language Processing (NLP), Bidirectional Encoder Representations from Transformer (BERT) and Computer Vision algorithms in media information analyzing (news, video content, social media) with a view of determining market changes, as well as to develop a software for automation the enterprise registration process.

\section{Author contributions}

Andrii Ivashchenko and Tetiana Ivashchenko - developed the idea, implemented Business Assistant model, prototype, tested it, designed Conclusions.

Igor Chornodid - conducted literature review, wrote introduction and searched for necessary statistics data.

\section{References}

ActiveTrades. (2020). https://www.activtrades.com/

Bawack, R. E., Wamba, S. F., \& Carillo, K. D. A. (2019). Artificial intelligence in practice: implications for information systems research. 26th Americas Conference on Information Systems. Cancun. https://www.researchgate.net/publication/333853703

Davenport, T. H., \& Ronanki, R. (2018). Artificial intelligence for the real world. Harvard Business Review, 96(1), 108-116. https://hbr.org/2018/01/artificial-intelligence-for-the-realworld

Etrade. (2020). Stocks. https://us.etrade.com/what-we-offer/ investment-choices/stocks

Fortune 500. (2019). Top US companies by total revenues for their respective fiscal years. https://fortune.com/fortune500/2019

Finlay, S. (2018). Using a predictive model to make decisions. In S. Finlay, Artificial intelligence and machine learning for business ( $3^{\text {rd }}$ ed.) (pp. 42-46). Relavistic.

Hanlon, K. (2015). 6 Factors to consider when choosing a business entity. http://www.lucerelegal.com/6-factors-when-choosinga-business-entity/

Inc. (2019). 5000 the most successful companies in America. https://www.inc.com/inc5000/2019/top-private-companies2019-inc5000.html

Investing.com. (2020). US wheat futures historical data. https:// www.investing.com/commodities/us-wheat-historical-data

Ivankin, A. (2018). Public financial statements: is it possible to get it. https://nv.ua/biz/experts/publichnaya-finansovaya-otchetnost-mozhno-li-ee-poluchit-2471351.html

Kashyap, P. (2018). Industrial applications of machine learning. In P. Kashyap, Machine learning for decision makers (pp. 189234). Apress. https://doi.org/10.1007/978-1-4842-2988-0_5 
Kenton, W. (2019). Small business administration (SBA). https:// www.investopedia.com/terms/s/small-business-administration.asp

Kopanakis, J. (2019, May 9). How artificial intelligence positively influences business decision making. https://www.mentionlytics.com/blog/how-artificial-intelligence-positively-influences-business-decision-making/

Liberto, D. (2019). Small and Mid-size Enterprises (SME). https:// www.investopedia.com/terms/s/smallandmidsizeenterprises. asp

Law of Ukraine. (2017). "About Amending the Law of Ukraine "About Accounting and Financial Reporting in Ukraine" regarding the improvement of certain provisions" dated 05.10.2017 No. 2164-VIII.

Michalewicz, Z., Schmidt, M., Michalewicz, M., \& Chiriac, C. (2006). Adaptive business intelligence. Springer. https://doi.org/10.1007/978-3-540-49774-5_8

Neortaite, J. \& Bulteris, R. (2009). Improving business rules management through the application of adaptive business intelligence technique. Information Technology and Control, 38(1), 21-28.

Our World in data. (2020). Food prices refer to the average price of particular food commodities globally and across countries. https://ourworldindata.org/food-prices

OANDA. (2020). Solutions for business, historical exchange rate. https://www1.oanda.com/fx-for-business/historical-rates

OECD. (2017). Technology and innovation in the insurance sector. OECD Publishing, Paris. https://www.oecd.org/finance/ Technology-and-innovation-in-the-insurance-sector.pdf

OECD. (2019). Artificial intelligence in society. OECD Publishing, Paris. http://doi.org/10.1787/eedfee77-en

Paliukas, V., \& Savaneviciene, A. (2018). Harmonization of rational and creative decisions in quality management using AI technologies. Economics and Business, 2018, 32, 195-208. https://doi.org/10.2478/eb-2018-0016

Phillips-Wren, G., \& Jain, L. (2006). Artificial intelligence for decision making. In Knowledge-based and intelligent information and engineering systems (pp. 531-536). Springer.

https://doi.org/10.1007/11893004_69
Ransbotham, S., Kiron, D., \& Gerbert, P., \& Reeves, M. (2017). Reshaping business with artificial intelligence: closing the gap between ambition and action. MIT Sloan Management Review, 59(1). http://image-src.bcg.com/Images/Reshaping\%20 Business\%20with\%20Artificial\%20Intelligence_tcm9-177882. pdf

Schatsky, D., Muraskin, C., \& Gurumurthy, R. (2014). Demystifying artificial intelligence: what business leaders need to know about cognitive technologies. A Deloitte Series on Cognitive Technologies. Deloitte University Press. https://www2.deloitte.com/content/dam/insights/us/articles/what-is-cognitive-technology/DUP_1030-Cognitive-Technologies_MASTER.pdf

Schatsky, D., Muraskin, C., \& Gurumurthy, R. (2015). Cognitive technologies: the real opportunities for business. Deloitte Review, 16, 115-129. https://www2.deloitte.com/us/en/insights/ deloitte-review/issue-16/cognitive-technologies-business-applications.html

Shefford, A., \& Holland, P. (2018). Trust in artificial intelligence. Transform Your Business with Confidence. https://assets.kpmg/ content/dam/kpmg/ph/pdf/services/TrustInArtificialIntelligence.pdf

Soumya, S. (2019). What are the factors that affect the choice for the form of organisation? http://www.preservearticles.com/ economics/what-are-the-factors-that-affect-the-choice-forthe-form-of-organisation/21247

The Billion Prices Project. (2019). Daily price indices, monthly, and annual inflation rates for Argentina and the US. http://www.thebillionpricesproject.com/datasets/

The Open Data of 500 US companies. (2020). http://www.opendata500.com/us/list/

US government. (2020). https://www.usa.gov

US Stocks. (2020). https://money.cnn.com/data/us_markets/

Voitenko, T. (2019). Graduation of enterprises according to the Ministry of Finance. Accounting Week. 1. https://i.factor.ua/ journals/bn/2019/january/issue-1/article-41682.html

Weller, C. (2017). Swedes can find out each other's salaries with just one phone call - but there's a catch. https://www.businessinsider.com/sweden-salaries-freely-available-2017-4 